\title{
IDLE MOTION SYNTHESIS OF HUMAN-LIKE THREE-DIMENSIONAL AGENT FOR THE HMI SYSTEMS
}

\author{
Maja Kocoń \\ West Pomeranian University of Technology, Szczecin \\ Sikorskiego 37, Szczecin, Poland
}

\begin{abstract}
This paper focuses on the problem of the synthesis of a virtual person's head movements while waiting for a reaction in human-machine interaction systems. The presented technique uses a 3D model of the human head, utilising a chain of rigid elements. We proposed an approach to perform an animation of a virtual head in idle mode. The idle mode is a state when a virtual person or a robot performs the subtle movements during the waiting phases between interaction intervals. Such type of action eliminates the problem of character "freezing" and has a higher level of acceptance to the user. This study covers issues connected with generating movements trajectories to rotate the rigid objects of the head model. The proposed approach has been exploited to perform smooth movements in the idle mode. Since the used algorithm has low computational requirements and introduces low delays in interaction loop, it can be used in HMI system working in real-time.
\end{abstract}

\section{KEYWORDS}

Idle Motion, Human-Computer Interaction (HMI), Head Motion, Virtual Human, Motion Synthesis

\section{INTRODUCTION}

In recent years, research on human-machine interaction (HMI) shows the growing popularity of the use of virtual reality (i.e. serious games, systems with human-like interfaces). Therefore, in HMI systems, communication between the user and the machine can be performed with the use of a virtual representation of a human, commonly known as an avatar. The main goal of HMI researchers is to design an interaction similar to interpersonal communication. Thanks to movable avatars, information can be transmitted using non-verbal signals (such as facial expressions, head movements and body language), which may provide intuitive interaction and improve communication.

The main channel of communication in non-verbal interaction is facial expressions and head gestures because they provide information about the emotional state or intentions of a person (Boker 2011). The face transmits a person's emotional state, but it does not adequately take into account the intensity of emotions (Cohn 2004) and the situational context. For this reason, it is crucial to consider the movement aspects of the head to interact fully. The meaning of the head motion in interaction is emphasized in many publications about social behaviours. Harrigan (2005) and Graf (2002) studied the effects of head movement in nonverbal communication. For example, the basic head actions such as tilt and node are essential in conversation, in active listening. Therefore head nodding can be used instead of verbal information like "yes"/ "no" (Boker 2011, Heylen 2005, Munhall 2004) or can be used for the point at something: "this one"/ "that one" (Tojo 2000). Furthermore, the sense of the spoken words is defined by the head movements (Sun 2011, Greenwood 2017).

In the works on the human motion, it can be noticed that most research concerns the expression of emotions, and little attention is paid to the concept of idle movements. In real life, people are always in motion, even when they don't do anything, but avatars and robots usually remain in standby mode. Because the main goal of this study is to model human behaviour, we should consider the situation when the virtual person is waiting for interactions with the user. Idle gestures are interpreted as subtle actions that appear when the avatar or robot is waiting for interactions with the environment and does not perform any task. These movements include head-swaying, small facial grimaces and eye blinking. This type of activities 
eliminates the moments when the avatar does not move (Kocon 2014) what appears many times in HCI applications or games. As a result, the realism of the animation increases, and the risk of the uncanny valley (MacDorman 2006) effect decreases. Many applications still ignore this issue, which results in unnatural behaviour for the human - steady position or repetitive sequence. The importance of using such gestures has been presented in (Salem 2011), where the comparison of two interactions schemes was presented. The gesture-based robot was perceived more positively by users because it displayed social skills.

Head gestures complete verbal communication and can improve the realism of the virtual character behaviour. Therefore, three-dimensional human is popular in HMI applications like social robotics (Ligthart 2018), driver assistance systems (Schwarz 2017) where we have user-friendly interfaces as personalized avatars, or in serious games (Cai 2018).

Character movement can be generated using many different methods. For this purpose, we can use data captured from a video sequence, audio signal or can be generated randomly. Idle aspects were described in (Jung 2013), where Jung et al. showed the importance of body motion in interactionless situations by testing five selected roles of movement used in cases when one does not speak, one of them are idling. In their researches, the anthropomorphic aspects of motion do not be included, and the aim goal was to find rules to create preferable motion scheme. In Egges (2004) based on Principal Component Analysis, they generate two layers of subtle motions like variations in body posture and change of balance when a person is standing. This approach is used to obtain the continuity between used animations without additional motion sequences. Based on a dedicated database of full body postures and different sequences of motion, idle animation is generated to represent a specific person.

\section{PROPOSED APPROACH}

The approach in this work can be used to provide idle motion in virtual space. Because the idle mode is not a primary movement in the interaction scheme and has an effect on continuously performed actions, it is desirable to use a technique with a low computational cost. Since for the humans the most readable form of information is the message communicated by another person, we decided to focus on motion synthesis using the human-like virtual character. In our visual representation of the head, we have taken the essential anthropometrical feature of the human face and head into consideration, what is present in Figure 1. We proposed a technique to perform a virtual person's head movements while waiting for a reaction with the generation of non-repetitive and unnatural gestures.

a)

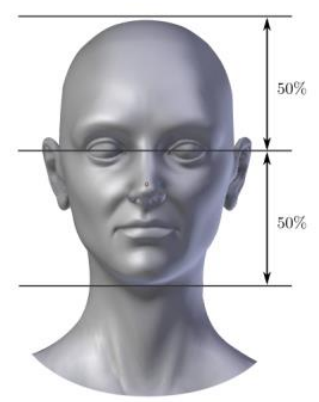

b)

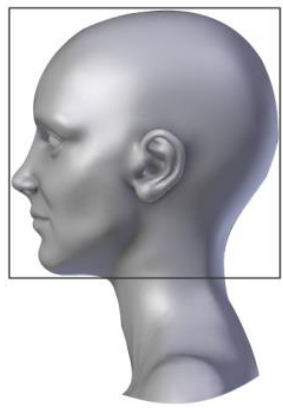

c)

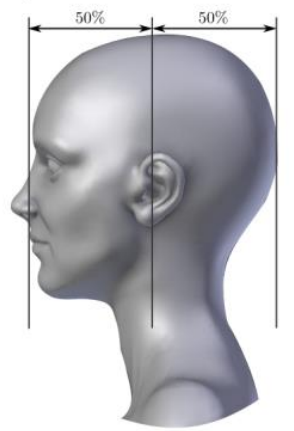

d)

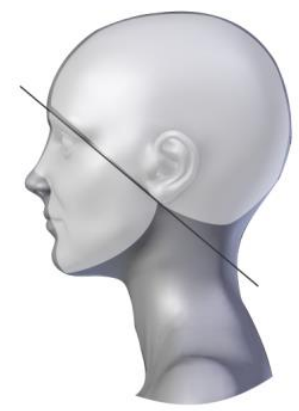

Figure 1. The main proportions of the head: (a) the distance between the jaw and the top of the head; (b) the head viewed in profile; (c) the position of the ear; (d) division of the head into a posterior- upper skull and facial part

\subsection{Human Head Motion}

Initial motion analysis was made on a test group of 20 people. Sequences were recorded in the front, side, and top view to obtaining ranges of motion for three rotations. Waiting for the interaction took from 15 to 30 seconds and a total of 180 sequences were obtained. Based on the recorded sequences, types of motion for three rotations in the idle situation were observed, what is shown in Figure 2. The ranges of motion obtained for selected types of rotation are presented in Table 1 . 

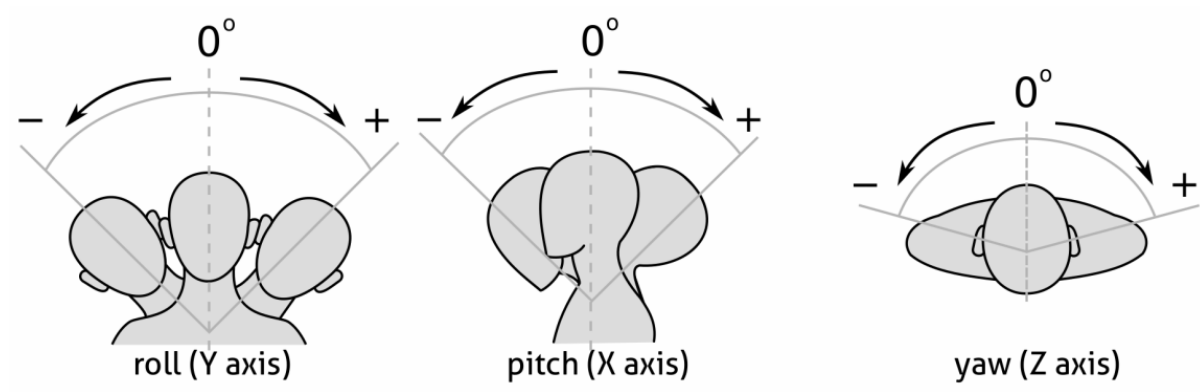

Figure 2. Selected gestures of the human head

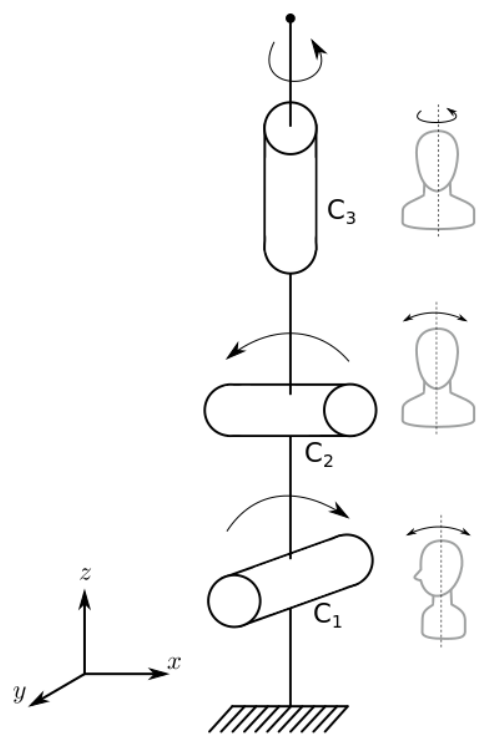

Figure 3. The chain of rigid elements defined for head movements

Table 1. The ranges of motion expressed in degrees for selected gestures of the human head

\begin{tabular}{|c|c|c|c|c|c|}
\hline \multicolumn{2}{|c|}{ Roll } & \multicolumn{2}{c|}{ Pitch } & \multicolumn{2}{c|}{ Yaw } \\
\hline Min & Max & Min & Max & Min & Max \\
\hline-14 & 19 & -12 & 20 & -23 & 32 \\
\hline
\end{tabular}

Head is analyzed as a rigid body; therefore, for motion description, we have used the set of non-deformable elements connected by joints. Based on the anatomical structure of the human head, we have selected three rigid elements that correspond to the head and neck. As you can see in Figure 3 is presented kinematic chain, where element $C_{1}$ indicate pitch motion, element $C_{2}$ roll rotation and element $C_{3}$ yaw motion. In our approach, three degrees of freedom was used because in basic model is more useful than more or less angles (Mukherjee 2015).

\subsection{Idle Motion Synthesis}

In idle mode, the subtle head gestures can be modelled by slight rotations of rigid elements. The movements are random for every axis in three-dimensional space. To generate such movements, we have decided to create rotation trajectories where every angle change is performed by correcting the actual angle by the trajectory value in each animation frame. For this purpose in this work, we propose a scheme to generate trajectories for modelling a human head model by rotating rigid elements using pseudo-random source. 
The procedure of trajectory generation can be summarised in the following steps:

1. Generate a sequence of numbers where any value $p(n)$ has to satisfy the condition $|p(n)-p(n-1)|=|p(n)-p(n+1)|=1$, where $n=1, \ldots, N-2$ and $N$ is the length of the trajectory.

2. Smoothing obtained trajectory by performing the convolution of the data with the smoothing window kernel.

3. Normalization and adjustment the smoothed trajectory to the expected range of changes.

As a pseudo-random source of numbers, we have decided to use a linear congruential generator (LCG) algorithm (Press 2007) due to its simplicity and ease of obtaining the same series of numbers for a given configuration. Such a generator can be described as:

$$
r(n+1)=[a \cdot r(n)+c] \bmod m,
$$

where $a, c$ and $m$ are constants which define the generated sequence of pseudo-random numbers, $n=$ $0, \ldots, N-1$ and $r(0)$ is the seed value. In our experiments, we have use $a=3101517285, c=672311$ and $m=2^{32}$ which have been selected during experiments. The values have no important meaning in this context but can be used to reproduce experiments.

The trajectory is generated using the formula:

$$
u(n+1)=\underbrace{[r(n+1) \bmod 3]-1}_{\delta}+u(n),
$$

where $\boldsymbol{u}(\mathbf{0})=\mathbf{0}, \boldsymbol{n}=\mathbf{0}, \ldots, \boldsymbol{N}-\mathbf{1}$ and modulus operation guarantee that changes between consecutive values can be equal to $\boldsymbol{\delta} \boldsymbol{\epsilon}\{-\mathbf{1}, \mathbf{0}, \mathbf{1}\}$ only. An example trajectory generated for $\boldsymbol{r}(\mathbf{0})=\mathbf{1 5 3}$ and $\boldsymbol{N}=\mathbf{9 0 0}$ is presented in Figure 4.

In the next step, smoothing is performed to remove rapid changes which are undesirable for natural-looking animation. The smoothing process has been carried out by using low-pass filtering with a smoothing window kernel (Smith 1997). The filtering operation is realized by convolution of the generated trajectory with kernel function (Press 2007):

$$
s(n)=\sum_{m=0}^{W-1} w(m) \cdot u(n-m),
$$

where: $W$ is the window size and $u(n-m)=0$ for $n-m<0$.

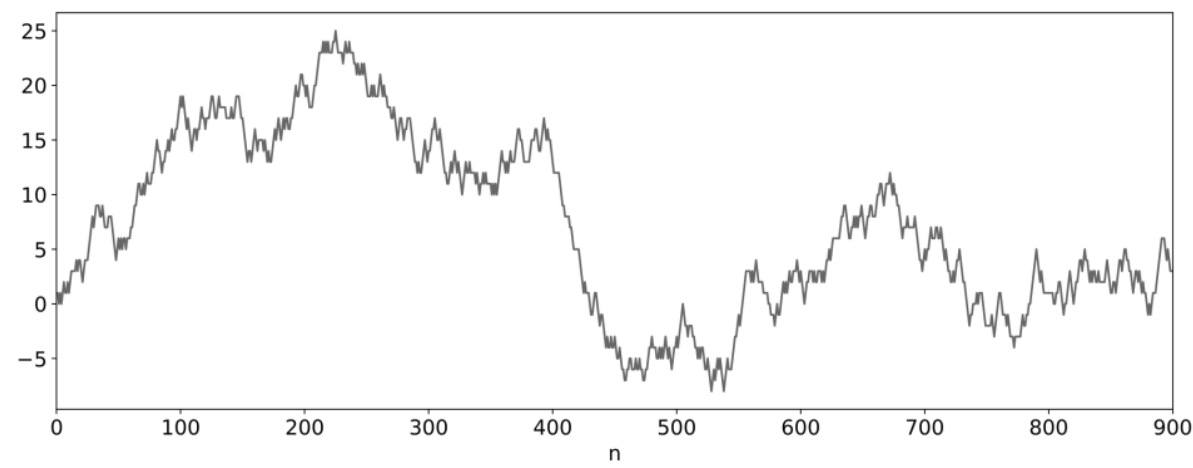

Figure 4. Example of the generated trajectory 
Many window functions can be used for smoothing, and the level of smoothness is mainly dependent on kernel size. The selection of the window is not significant for the considered application, so we decided in our study to use the Hanning window defined as (Poularikas 1998):

$$
w(m)=\frac{1}{2} \cdot\left[1-\cos \left(\frac{2 m \pi}{W}\right)\right]
$$

where: $m=0, \ldots, W-1$. The result of the smoothing stage for the generated signal (Figure 4 ) is depicted in Figure 5.

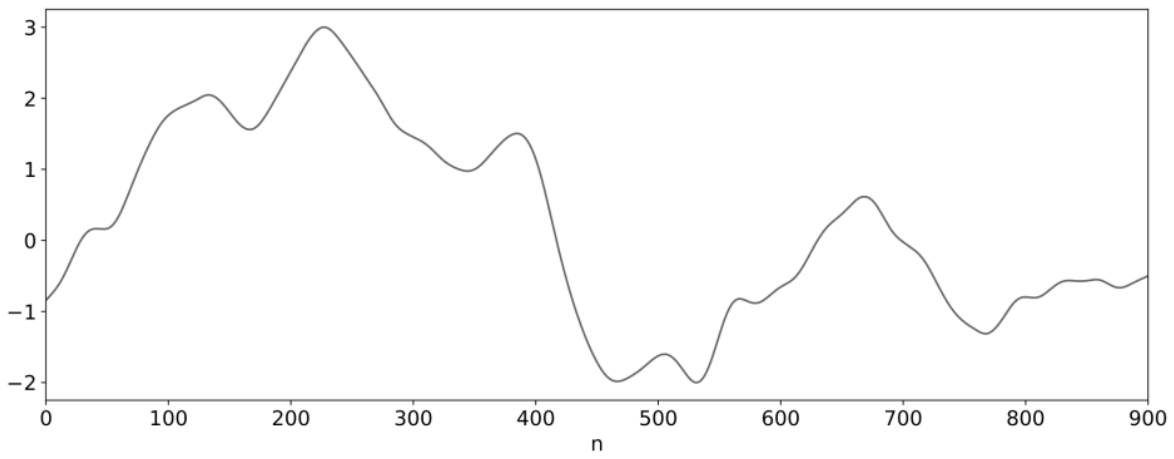

Figure 5. Example trajectory after applying smoothing and normalization operations

In the last phase, the smoothed trajectory is adjusted to the desired range change where all values should be in the range $\left[\alpha_{1}, \alpha_{2}\right]$ and $\alpha_{1}<\alpha_{2}$. Therefore, a new stage has been applied where the smoothed data is scaled and adjusted to the final range:

$$
\widehat{u}(n)=\alpha_{1}+\frac{s(n)-s_{\min }}{s_{\max }-s_{\min }} \cdot\left(\alpha_{2}-\alpha_{1}\right),
$$

where $s_{\min }$ and $s_{\max }$ denotes the minimal and maximal value of $s(n)$ respectively.

As an example of a final $\hat{u}(\mathrm{n})$ trajectories for head animation in idle mode, trajectories generated for each rigid object are presented in Figure 6, where the parameters are shown in Table 2.

Table 2. Parameters of example trajectories.

\begin{tabular}{|c|c|c|c|c|}
\hline $\boldsymbol{N}$ & $\boldsymbol{W}$ & $\boldsymbol{r}(\mathbf{0})$ & $\boldsymbol{\alpha}_{\mathbf{1}}$ & $\boldsymbol{\alpha}_{\mathbf{2}}$ \\
\hline 900 & 59 & 27 & -4 & 12 \\
900 & 59 & 37 & -13 & 13 \\
900 & 59 & 12 & -9 & 9 \\
\hline
\end{tabular}




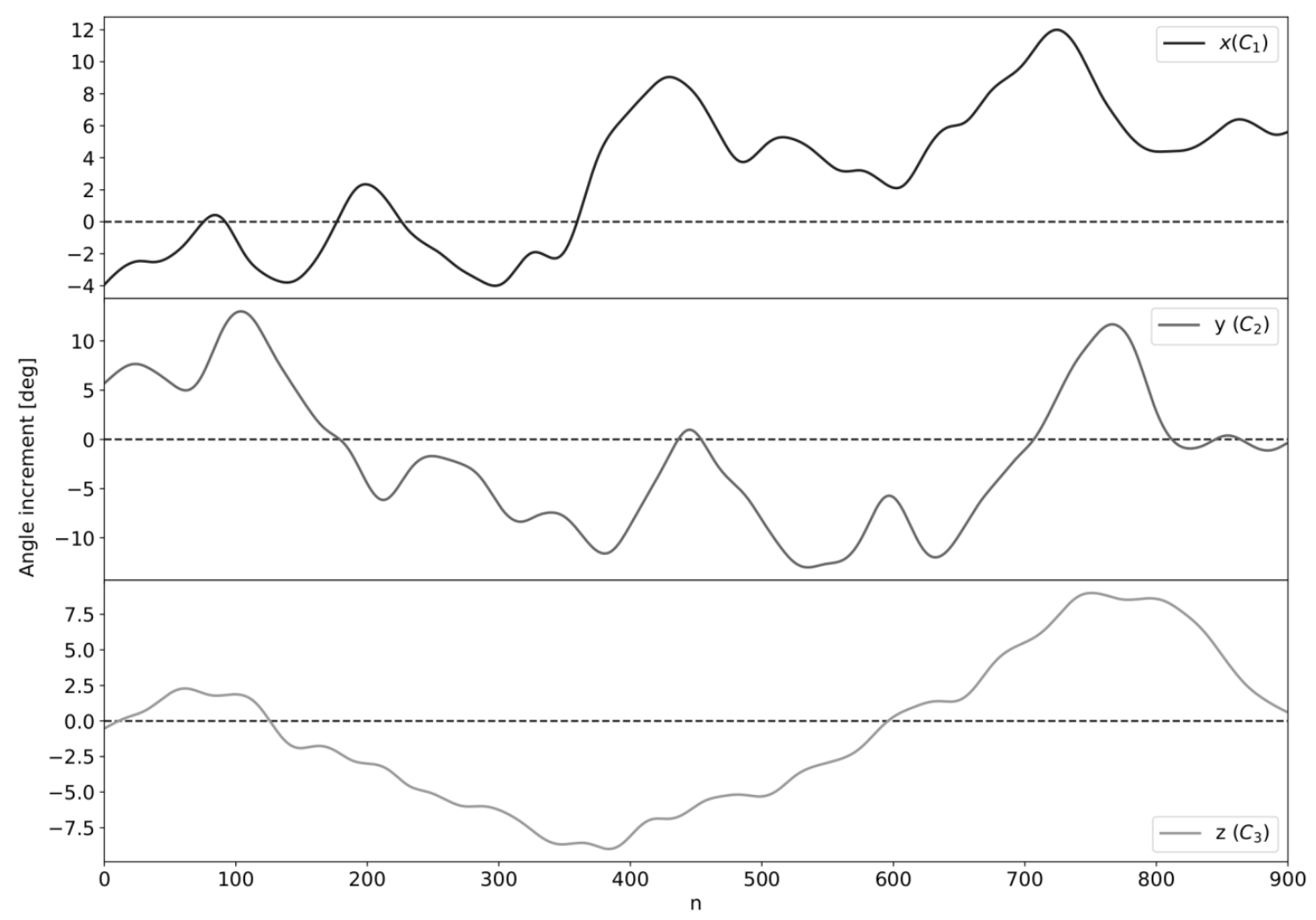

Figure 6. Example generated trajectories for each rigid object in idle mode

The advantages of the proposed approach include the simplicity, low computational cost and reproducibility of trajectories for a given length $(\boldsymbol{N})$ and random seed $(\boldsymbol{r}(\mathbf{0}))$. The configuration parameters allow us to get a whole range of functions for animation process modelling.

Finally, based on the proposed approach, we have obtained motion sequences for idle mode, an example of a few frames of animation is shown in Figure 7.

\section{CONCLUSION AND FUTURE WORKS}

The approach of modelling head movements of artificial agents in the state between interactions is presented in this work. The increasing popularity of HMI systems stimulates the interest in developing techniques to precise modelling movements of avatars. The main problem is connected with behaviour modelling in the case when the interaction is held in the absence of external events. The method presented in this study allows for effective simulation of subtle head motions by incorporating the motion trajectories for angle modification of rigid bodies used to move the model of the human head. The proposed algorithm exploits the simple generator, smoothing and normalizing stages to obtain final trajectories. The resulting movements of the head are steady and can be used directly in the HMI system. In future work, we plan to design a mechanism to generate seamless trajectories and its morphing to simplify the process of subtle movements dedicated to idle mode. 

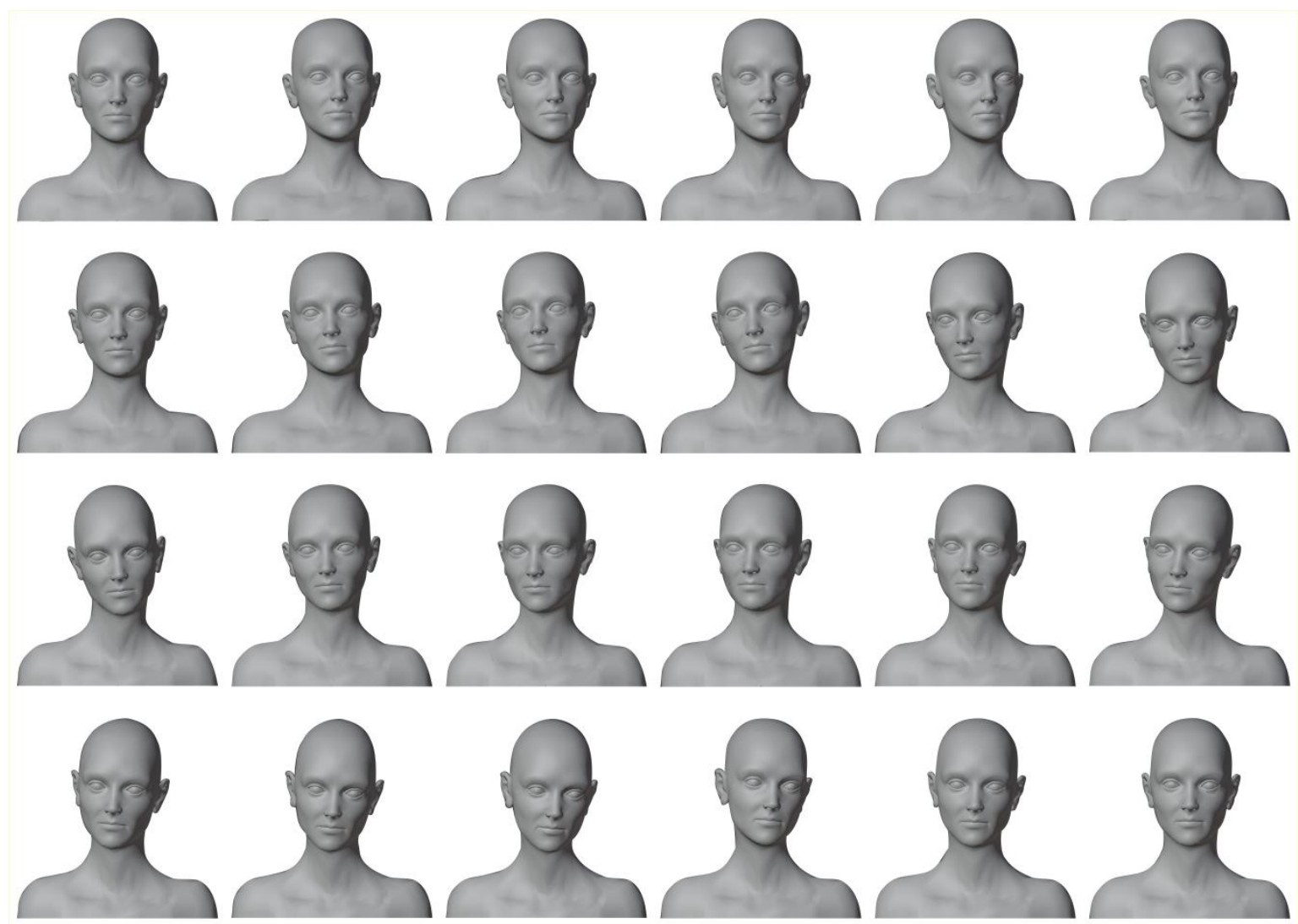

Figure 7. Example animation frames for the person in idle mode

\section{REFERENCES}

Boker, S. et al, 2011. Something in the Way We Move: Motion Dynamics, Not Perceived Sex, Influence Head Movements in Conversation. Journal of experimental psychology. Human perception and performance, Vol. 37, pp. 874-91.

Cai, Y. et al, 2018. VR, Simulations and Serious Games for Education (1st. ed.). Springer Publishing Company, Incorporated.

Cohn, J. F. et al, 2004. Multimodal coordination of facial action, head rotation, and eye motion during spontaneous smiles. Sixth IEEE International Conference on Automatic Face and Gesture Recognition, Seoul, Korea, pp. 129-138.

Egges, A. et al, 2004. Personalised Real-Time Idle Motion Synthesis. 12th Pacific Conference on Computer Graphics and Applications (PG 2004), Seoul, Korea, pp. 121-130.

Graf, H. P. et al, 2002, Visual prosody: facial movements accompanying speech. Proceedings of Fifth IEEE International Conference on Automatic Face Gesture Recognition, Washington, DC, USA, pp. 396-401.

Greenwood, D. et al, 2017. Predicting Head Pose from Speech with a Conditional Variational Autoencoder. Proceedings of Interspeech 2017, ISCA, pp. 3991-3995.

Harrigan, J. et al, 2005. The New Handbook of Methods in Nonverbal Behavior Research. Oxford University Press, Series in Affective Science.

Heylen, D, 2005. Challenges Ahead: Head movements and other social acts during conversations. Proceedings of the Joint Symposium on Virtual Social Agents. The Society for the Study of Artificial Intelligence and the Simulation of Behaviour, pp. 45-52.

Jung, J., et al, 2013. Guidelines for Contextual Motion Design of a Humanoid Robot. Int J of Soc Robotics, Vol.5, pp. 153-169. 
Kocoń, M, 2014. Facial expressions modeling for interactive virtual environments. 19th International Conference on Methods and Models in Automation and Robotics, Miedzyzdroje, Poland, pp. 744-747.

Ligthart, M. et al, 2018. Reducing Stress by Bonding with a Social Robot: Towards Autonomous Long-Term Child- Robot Interaction. ACM/IEEE International Conference on Human-Robot Interaction, Chicago, United States.

Macdorman K., F., 2006. Subjective Ratings of Robot Video Clips for Human Likeness, Familiarity, and Eeriness: An Exploration of the Uncanny Valley. ICCS/CogSci-2006 Long Symposium: Toward Social Mechanisms of Android Science.

Mukherjee, S. S. and Robertson, N. M., 2015. Deep Head Pose: Gaze-Direction Estimation in Multimodal Video. IEEE Transactions on Multimedia, Vol. 17, No. 11, pp. 2094-2107.

Munhall, K.G. et al, 2004. Visual Prosody and Speech Intelligibility Head Movement Improves Auditory Speech Perception. Psychological science, Vol. 15, pp. 133-137.

Poularikas, A., 1998. Handbook of Formulas and Tables for Signal Processing. Electrical Engineering Handbook. Springer Berlin Heidelberg.

Press,W. H. et al, 2007. Numerical Recipes 3rd Edition: The Art of Scientific Computing. Cambridge University Press, USA, 3 edition.

Salem, M. et al, 2011. Effects of Gesture on the Perception of Psychological Anthropomorphism: A Case Study with a Humanoid Robot. Social Robotics. ICSR 2011, Lecture Notes in Computer Science, Vol. 7072, Springer, Berlin, Heidelberg.

Schwarz, A., 2017. DriveAHead - A Large-Scale Driver Head Pose Dataset. IEEE Conference on Computer Vision and Pattern Recognition Workshops (CVPRW), Honolulu, pp. 1165-1174.

Smith, S. W., 1997. The Scientist and Engineer's Guide to Digital Signal Processing. California Technical Publishing, USA.

Sun, $\mathrm{X}$ et al, 2011.Towards visual and vocal mimicry recognition in human-human interactions. 2011 IEEE International Conference on Systems, Man, and Cybernetics, Anchorage, AK, pp. 367-373.

Tojo, T. et al, 2000. A conversational robot utilizing facial and body expressions. 2000 IEEE international conference on systems, man and cybernetics, Nashville, TN, Vol. 2, pp. 858-863. 